\title{
Delay and state observation for SISO nonlinear systems with input delay
}

\author{
Vincent Léchappé, Jesus De Leon, Emmanuel Moulay, Franck Plestan, Alain
} Glumineau

\section{- To cite this version:}

Vincent Léchappé, Jesus De Leon, Emmanuel Moulay, Franck Plestan, Alain Glumineau. Delay and state observation for SISO nonlinear systems with input delay. International Journal of Robust and Nonlinear Control, Wiley, 2018, 28 (6), pp.2356-2368. <10.1002/rnc.4020>. <hal-01668933>

\section{HAL Id: hal-01668933 \\ https://hal.archives-ouvertes.fr/hal-01668933}

Submitted on 20 Dec 2017

HAL is a multi-disciplinary open access archive for the deposit and dissemination of scientific research documents, whether they are published or not. The documents may come from teaching and research institutions in France or abroad, or from public or private research centers.
L'archive ouverte pluridisciplinaire HAL, est destinée au dépôt et à la diffusion de documents scientifiques de niveau recherche, publiés ou non, émanant des établissements d'enseignement et de recherche français ou étrangers, des laboratoires publics ou privés. 


\title{
Delay and state observation for SISO nonlinear systems with input delay
}

\author{
Vincent Léchappé ${ }^{1 *}$, Jesús de León ${ }^{2}$, Emmanuel Moulay ${ }^{3}$, Franck Plestan ${ }^{4}$, Alain \\ Glumineau $^{4}$ \\ ${ }^{1}$ Griffith university, School of Engineering, Gold Coast, Australia \\ ${ }^{2}$ FIME, Universidad Autónoma de Nuevo León, Monterrey, Mexico \\ ${ }^{3}$ XLIM, UMR CNRS 7252, Université de Poitiers, Poitiers, France \\ ${ }^{4}$ Ecole Centrale de Nantes - LS2N, UMR CNRS 6004, Nantes, France
}

\begin{abstract}
SUMMARY
This paper deals with the problem of state and delay estimation for SISO nonlinear systems with an unknown time-varying delay in the input. The main idea is to approximate the delayed input by using Taylor's theorem and to create an extended system with the delay as part of the extended state. Then, the construction of an observer is proposed to estimate both state and delay. The results are illustrated by simulations. Copyright (c) 0000 John Wiley \& Sons, Ltd.

Received ...
\end{abstract}

KEY WORDS: Input delay; Time-delay estimation; High-gain observer.

\section{INTRODUCTION}

Input-delay systems are a subcategory of time-delay systems (TDS). They especially include all remote controlled devices. The source of delay is multiple: the network configuration (see the extensive literature on Networked Control Systems [1]), computational delays or physical transport delays. When the delay is small, delay free controllers can often achieve stabilization. However, predictive techniques are often required as soon as the delay becomes larger and cannot be neglected anymore [2]. To use such methods, the value of the delay is needed. However, in real applications, it is quite difficult to measure the delay with precision that is why it can be necessary to estimate it and compute a prediction based on this estimated delay. For an exhaustive review of time delay estimation (TDE) techniques, the reader can refer to the report [3]. The prediction requires not only

*Correspondence to: Griffith university, School of Engineering, Gold Coast, Australia. E-mail: lechappe.vincent@gmail.com

Contract/grant sponsor: This work was supported by the bilateral project Mexico-Francia (PCP 2013) between the Ecole Centrale de Nantes and the Universidad Autónoma de Nuevo León.; contract/grant number: 209488 CONACYT

Copyright (c) 0000 John Wiley \& Sons, Ltd.

Prepared using rncauth.cls [Version: 2010/03/27 v2.00] 
the delay, but also the state. However, standard observation techniques cannot be applied when the delay is unknown. In this paper, both problems are addressed: delay estimation and state observation.

\subsection{Delay estimation}

Signal processing approach. Time delay identification has often been based on a signal processing approach and particularly in the acoustic field [4][5]. In this case, the general idea of TDE techniques is the following: an estimator $J(\hat{\tau})$ is designed ${ }^{\dagger}$ and the evolution law for $\hat{\tau}$ has to minimize the criterion $J(\hat{\tau})$. A standard time delay estimator is the direct correlator (DC) that seeks the maximum value of the cross correlation between the reference and the delayed signal [5]. Other estimators exist such as $J(\hat{\tau})=[u(t-\tau)-u(t-\hat{\tau})]^{2}$ (where $u$ is the system input) or the average square difference function estimator (ASDF) for discrete time techniques [6]. These methods are not well adapted in the control context for two main reasons. First of all, they are usually offline methods (DC, ASDF) and therefore suffer from a long computation time due to the low rate of convergence of the optimization algorithm. Secondly, they require the knowledge of the delayed signal which is not always available in practice. A survey of TDE techniques with a signal processing focus is given in [7].

Control oriented methods. In these approaches, the delay is often considered as a parameter of the system and its identification is usually combined with the identification of other parameters. A vast literature exists on this topic. Some authors use the frequency domain where the delay appears as a parameter in the exponential $e^{-\tau s}$. In [8], the term $e^{-\tau s}$ is approximated by a rational transfer function of the Padé form; then a standard discrete least-square algorithm is used to minimize an objective function. Tuch et al. [9] also based their approach on the frequency domain and proposed a continuous recursive least square algorithm. However, this method does not work if the initial conditions of the system are not perfectly known. In [10], a PDE approximation is used to extract the delay. In [11], a similar techniques as in [9] is applied but the value of $u(t-\tau)$ is required. In [12], observers have been used to identify the delay. However, all the state and its time derivatives are needed; so the method is very sensitive to noise measurement. In [13] and [14], a convolution approach is discussed for transfer function systems. Finally, recent works design adaptive controllers that estimate the delay. Unlike previously mentioned papers, next references deal with closed-loop identification method. In [15], the delay estimator is obtained from the transport PDE representation of the system. However, only the state stabilization is proven but not the convergence of the delay estimator. A delay identification law is proposed in [16] for sampled systems. This law is based on a particular form of systems with two distinct real eigenvalues of multiplicity one. Note that, in all previously mentioned articles except [16], the delay is constant.

\subsection{State observation with unknown delay}

The article [17] provides a survey on state observation in presence of a constant delay. As mentioned in [18], most of the works deal with state observation in presence of a known delay: see for example [19] for output delay and [20] for state and input delays. Few articles deal with the unknown delay case. In [18], a high-gain observer guarantees the practical stability of the observation error. In [21],

${ }_{\dagger}^{\dagger}$ The delay is often denoted by the letter $h$ or $\tau$. In this paper, the notation $\tau$ will be used. 
the observation is coupled with the identification of some parameters of the system. In [18] and [21], the delay is unknown but it is not estimated.

\subsection{State observation and delay estimation}

As mentioned in [22], the problems of state and delay estimation are much easier to solve separately because the knowledge of the state will help to estimate the delay and vice-versa. That is why, estimating both the delay and the state will allow to achieve more accurate estimations (instead of considering the delay as a perturbation like in [18]). As far as the authors knowledge, very few articles deal with both delay identification and state reconstruction. One of them is [16] where the design of the state observer is largely based on a particular sampling/holding technique. In [23], the authors introduced an extended observer for SISO LTI systems that can observe both state and delay but it suffers from an important observation singularity. In [24], an interconnected observation scheme is proposed to observe both the delay and the state. However, the knowledge of the retarded input is required. A joint state and delay estimation techniques is presented in [22] for nonlinear systems with piecewise constant state delay.

\subsection{Contribution}

The main contribution of this article is to offer an online estimation method, based on observation theory, for both state and delay values. The method works for time-varying delays and requires the knowledge of the input value, its derivatives and the output at time $t$. This work is focused on the estimation of a single time-varying delay in the input of SISO nonlinear systems; no parameter identification is considered. It is an extension of [23] to nonlinear systems that allows to circumvent the observation singularity problem and to improve the accuracy of the estimation. Note that, only open-loop results are presented in this paper.

\subsection{Paper's structure}

The paper is organized as follows. The problem presentation and an observability study are provided in Section 2. Section 3 is dedicated to the observer design and the convergence analysis: the general case with the knowledge of $m$ time-derivatives of the input is worked out. In Section 4, it is illustrated in simulation that the knowledge of the first two time-derivatives is enough to achieve accurate estimations. Conclusions and future developments are given in Section 5.

\subsection{Notations and definitions}

The matrix $I_{n}$ is the identity matrix of dimension $n$. The vector $0_{n \times 1}$ stands for a column vector of dimension $n$ whose all components are null. The matrix inequality $A<B$ (resp. $A>B$ ) means that $A-B$ is negative definite (resp. positive definite). The entry in the $i^{\text {th }}$ row and $j^{\text {th }}$ column of a matrix $A$ is referred to as $A_{(i, j)}$. The vector norm and matrix norm $\|$.$\| are respectively the$ Euclidean norm and the spectral matrix norm. The function $u \in C^{m+1}\left(\left[-\tau_{\max },+\infty[, \mathbb{R})\right.\right.$ means that $u:\left[-\tau_{\max },+\infty[\rightarrow \mathbb{R}\right.$ has $m+1$ continuous derivatives. A continuous function $\beta:[0, a[\rightarrow[0,+\infty[$ is said to belong to class $\mathcal{K}$ if it is strictly increasing and $\beta(0)=0$. The notation $u^{(i)}$ refers to the $i^{t h}$ derivative of $u$ with respect to time. When no confusion is possible, the variable 
$t$ will be omitted; for instance $x=x(t)$.

\section{PROBLEM STATEMENT AND OBSERVABILITY ANALYSIS}

\subsection{Problem statement}

The considered systems are SISO nonlinear systems with a time-varying delay $\tau(t)$ acting on the control input $u$. More precisely, the class of systems is defined by

$$
\left\{\begin{array}{l}
\dot{x}=\wedge x+f(x)+g(y) u(t-\tau(t)) \\
y=C x=x_{1} \\
x(0)=x_{0} \\
u(\theta)=\phi_{u}(\theta) \quad \forall \theta \in\left[-\tau_{\max }, 0\right]
\end{array}\right.
$$

where $\tau_{\max }$ is the maximal bound of the delay, $x=\left[x_{1}, \ldots, x_{n}\right]^{T} \in \mathbb{R}^{n}, y \in \mathbb{R}, u \in \mathbb{R}$, $I_{n}$ is the identity matrix of dimension $n, g(y)=[0, \ldots, 0, G(y)]^{T}, C=[1,0, \ldots, 0]^{T}$, $f(x)=\left[f_{1}\left(x_{1}\right), f_{2}\left(x_{1}, x_{2}\right), \ldots, f_{n}\left(x_{1}, \ldots, x_{n}\right)\right]^{T}$,

$$
\Lambda=\left[\begin{array}{cc}
0_{n \times 1} & I_{n-1} \\
0 & 0_{1 \times n}
\end{array}\right] .
$$

Note that system (1) is an upper-triangular system and a large class of observable systems can be rewritten in this form [25].

\section{Assumption 1}

The functions $f$ and $G$ are respectively in $C^{1}\left(\mathbb{R}^{n}, \mathbb{R}^{n}\right)$ and $C^{1}(\mathbb{R}, \mathbb{R})$ and $f$ is globally Lipschitz, i.e. there exists $c_{1}>0$ such that for all $(x, z) \in \mathbb{R}^{n} \times \mathbb{R}^{n}$

$$
\|f(x)-f(z)\| \leq c_{1}\|x-z\| .
$$

\section{Assumption 2}

The function $G$ is bounded, i.e. there exists $\bar{G}>0$ such that for all $y \in \mathbb{R}$

$$
|G(y)| \leq \bar{G}
$$

\section{Assumption 3}

The delay function is continuous, differentiable and verifies $\tau(t) \in\left[\tau_{\min }, \tau_{\max }\right]$. Its dynamics, denoted $\dot{\tau}(t)=\eta(t)$, is unknown and bounded

$$
|\eta(t)| \leq \bar{\eta}
$$

\section{Assumption 4}

Let $m \in \mathbb{N}^{*}$, the input $u$ is in $C^{m+1}\left(\left[-\tau_{\max },+\infty[, \mathbb{R})\right.\right.$ and its derivatives are known and bounded, i.e. for all $i=1, \ldots, m+1$, there exist constants $U_{i}>0$ such that for all 
$t \geq-\tau_{\max }$

$$
\left|u^{(i)}(t)\right| \leq U_{i}
$$

\section{Remark 2.1}

For completeness, the general case with $m \in \mathbb{N}^{*}$ is presented but for practical reasons that will be explained in the next sections, $m$ will be usually taken equal to 2 .

The objective is to design an observer that reconstructs the state $x(t)$ and the delay $\tau(t)$ from the knowledge of the output $y(t)$, the input $u(t)$ and its time derivatives. Usually, the delay is considered as a parameter of the system when it is constant [8], [26]. However, $\tau$ is time-varying in this work so the idea is to treat it as a state of an extended system. Denoting $X=\left[x^{T} \tau\right]^{T}$, one obtains

$$
\left\{\begin{array}{l}
\dot{X}=\tilde{f}(X, u) \\
y=Y(X)=C_{e x t} X=x_{1}
\end{array}\right.
$$

with $\tilde{f}(X, u)=\left(\begin{array}{c}\Lambda x+f(x)+g(y) u(t-\tau(t)) \\ \eta(t)\end{array}\right)$ and $C_{e x t}=[C, 0]$. Systems (1) and (2) are equivalent in the sense that they have the same state trajectories. In the next subsection, the observability of the augmented system (2) is going to be worked out.

\subsection{Delay observability}

In [23], the observability analysis has been performed on an approximated system whereas here it is done on the exact system (2). The concept of observability rank condition used in the next theorem is detailed in [27]. This concept is very convenient to check the observability of nonlinear systems this is why it will be used in the observability analysis performed thereafter. The following result is achieved.

\section{Theorem 1}

System (2) is rank-observable if and only if

$$
G(y(t)) \neq 0 \quad \forall t \geq 0
$$

and

$$
\dot{u}(t) \neq 0 \quad \forall t \geq-\tau_{\max } .
$$

\section{Proof}

The $n$ first time derivatives of the output $y$ read as

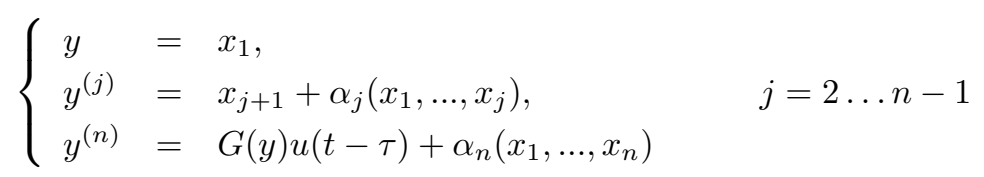


where the $\alpha_{j}$ are combinations of the $x_{i}$. Then, the Jacobian matrix of $y$ and its time derivatives has the following lower triangular form

$$
\frac{\partial\left(y, \dot{y}, \ldots, y^{(n)}\right)}{\partial X}=\left[\begin{array}{ccccc}
1 & 0 & 0 & \cdots & 0 \\
* & 1 & 0 & \cdots & 0 \\
\vdots & \ddots & \ddots & \ddots & \vdots \\
* & \cdots & * & 1 & 0 \\
* & \cdots & \cdots & * & G(y) \frac{\partial u(t-\tau)}{\partial \tau}
\end{array}\right]
$$

Therefore, system (2) satisfies the rank observability condition

$$
\operatorname{rank}\left[\frac{\partial\left(y, \dot{y}, \ldots, y^{(n)}\right)}{\partial X}\right]=n+1
$$

if and only if $G(y) \frac{\partial u(t-\tau)}{\partial \tau}$ does not cancel. In addition, for all $t \geq 0$ and $\tau \in$ $\left[\tau_{\min }, \tau_{\max }\right]$, one has

$$
\frac{\partial u(t-\tau)}{\partial \tau}=-\frac{\partial u(t-\tau)}{\partial t} .
$$

Then, $\frac{\partial u(t-\tau)}{\partial \tau} \neq 0$ is equivalent to $\frac{\partial u(t-\tau)}{\partial t} \neq 0$. This inequality should be verified for all $t \geq 0$ and $\tau \in\left[\tau_{\min }, \tau_{\max }\right]$ which is equivalent to (4) since the delay is continuous according to Assumption 3. This ends the proof.

Condition (4) is quite natural because if the input is constant then the delay has no influence on the system and it cannot be observed. To avoid this singularity $\dot{u}(t)=0$, it is assumed as in [11] that:

\section{Assumption 5}

There are time intervals $I$ where the first derivative of $u$ is continuous and bounded, i.e. for all $t \in I$,

$$
|\dot{u}(t)|>\alpha^{\prime}
$$

with $\alpha^{\prime}>0$.

Indeed, if (5) holds then (4) is automatically verified on $I$.

\section{A NEW SCHEME OF DELAY-STATE OBSERVER}

\subsection{Transformation of the extended system (2)}

System (2) is not in an adequate form to observe $\tau$. Therefore, the Taylor's theorem is used to take the delay out of the input. From Assumption 4, the input is $m$-times differentiable. Then, there exists a function $\gamma_{m}:\left[-\tau_{\max } ;+\infty\left[\times\left[-\tau_{\max },-\tau_{\min }\right] \rightarrow \mathbb{R}\right.\right.$ such that

$$
u(t-\tau(t))=\sum_{i=0}^{m} \frac{(-1)^{i} \tau(t)^{i}}{i !} u^{(i)}(t)+\gamma_{m}(t,-\tau(t))
$$


for all $t>-\tau_{\max }$ and $\gamma_{m}$ is called the remainder. From Assumption 4, since $u$ is $m+1$ differentiable the remainder $\gamma_{m}$ is such that

$$
\left|\gamma_{m}(t,-\tau(t))\right| \leq \frac{\tau(t)^{m+1}}{(m+1) !} U_{m+1} \leq \frac{\tau_{m a x}^{m+1}}{(m+1) !} U_{m+1} .
$$

In the sequel $m$ will be taken larger or equal to one because for $m=0$ the approximation (6) does not depend on $\tau$. By substituting (6) in (2), the following system is obtained

$$
\left\{\begin{aligned}
\dot{X} & =\Lambda_{e x t}(\xi(t)) X+f_{e x t}(X)+g_{e x t}(y) u(t)+\Gamma(t, y, \tau) \\
y & =C_{e x t} X=x_{1}
\end{aligned}\right.
$$

with $\quad X=\left[x^{T}, \tau\right]^{T}, \quad f_{\text {ext }}(X)=\left[f(x)^{T}, 0\right]^{T}, \quad g_{\text {ext }}(y)=\left[g(y)^{T}, 0\right]^{T}, \quad \Gamma(t, y, \tau)=$ $\left[g(y)^{T} \gamma_{m}(t,-\tau(t)), \eta(t)\right]^{T}$,

$$
\Lambda_{e x t}(\xi(t))=\left[\begin{array}{ccc}
0 & I_{n-1} & 0_{n-1 \times 1} \\
0 & 0_{1 \times n-1} & \xi(t) \\
0 & 0_{1 \times n-1} & 0
\end{array}\right]
$$

and

$$
\xi(t)=G(y) \sum_{i=1}^{m} \frac{(-1)^{i} \tau^{i-1}(t)}{i !} u^{(i)}(t)
$$

\section{Remark 3.1}

The use of the Taylor approximation (6) requires the computations of the input time derivatives. In this section, the case of a $m^{t h}$ order Taylor approximation is presented to deal with the general case. However, choosing $m=2$ is usually enough to get an accurate estimation with a limited number of input time-derivatives. In other words, from the engineering point of view, $m=2$ is the best choice to compute an accurate delay estimation and using only two input derivatives. This will be illustrated in Section 4.

Extended system (8) is equivalent to initial system (1) in the sense that both systems exhibit the same trajectories. However, system (8) is delay-free, with respect to the input, thanks to (6). The delayed nature of the plant is still present through $\gamma_{m}(t,-\tau(t))$ in $\Gamma$. The transformation is a convenient way to rewrite the system in order to apply existing results from the observers literature. The error of approximation $\gamma_{m}$ is going to be considered as a perturbation in the design of the observer as well as the dynamics of the delay $\eta(t)$. This is a key point of the method: considering the delay dynamics as a perturbation and designing an observer that is able to reconstruct the system state in spite of uncertainty $\Gamma$. Indeed, it is often a hard task to find a good approximation of the delay dynamics.

\subsection{Observer design}

Because of the form of (8) and because it is has only one tuning parameter, a high gain observer has been chosen (see [28], [29], [30] for further details on high gain observers). 
Let $\lambda(\xi) \in \mathbb{R}^{(n+1) \times(n+1)}$ and $\bar{A} \in \mathbb{R}^{(n+1) \times(n+1)}$ be

$$
\lambda(\xi)=\left[\begin{array}{cc}
I_{n} & 0_{n \times 1} \\
0_{1 \times n} & \xi
\end{array}\right] \quad \bar{A}=\left[\begin{array}{cc}
0_{n \times 1} & I_{n} \\
0 & 0_{1 \times n}
\end{array}\right] .
$$

Then, the following observer is designed:

$$
\left\{\begin{array}{l}
\dot{\hat{X}}=\hat{\Lambda}_{e x t} \hat{X}+f_{e x t}(\hat{X})+g_{e x t}(y) u(t)-\lambda^{-1}(\hat{\xi}) S_{\rho}^{-1} C_{e x t}^{T}\left(C_{e x t} \hat{X}-y\right) \\
\rho S_{\rho}+\bar{A}^{T} S_{\rho}+S_{\rho} \bar{A}-C_{e x t}^{T} C_{e x t}=0 \\
\dot{\hat{\tau}}_{p}=\operatorname{proj}_{\left[\tau_{\text {min }}, \tau_{\text {max }}\right]}(\dot{\hat{\tau}})
\end{array}\right.
$$

where $\hat{X}=\left[\hat{x}^{T}, \hat{\tau}\right]^{T} \in \mathbb{R}^{n+1}$ and $\hat{\Lambda}_{\text {ext }}=\Lambda_{\text {ext }}(\hat{\xi}(t)) \in \mathbb{R}^{(n+1) \times(n+1)}$ with

$$
\hat{\xi}(t)=G(y) \sum_{i=1}^{m} \frac{(-1)^{i} \hat{\tau}^{i-1}}{i !} u^{(i)}(t)
$$

and $\hat{\tau}$ is the estimated delay. It is reminded that $y=x_{1}$. Note that $\dot{\hat{\tau}}$ is computed from the first equation of system (9) and its projection, denoted $\dot{\hat{\tau}}_{p}$, is defined by the last equation of (9). The projection operator is given by

$$
\operatorname{proj}_{\left[\tau_{\min }, \tau_{\max }\right]}(\dot{\hat{\tau}})= \begin{cases}0, & \hat{\tau}=\tau_{\max } \text { and } \dot{\hat{\tau}}>0 \\ 0, & \hat{\tau}=\tau_{\min } \text { and } \dot{\hat{\tau}}<0 \\ \dot{\hat{\tau}} & \text { else. }\end{cases}
$$

One has the following result for the projection operator (11).

\section{Lemma 1}

If the initial condition is such that $\hat{\tau}(0) \in\left[\tau_{\text {min }}, \tau_{\text {max }}\right]$ and $\dot{\hat{\tau}}_{p}=\operatorname{proj}_{\left[\tau_{\text {min }}, \tau_{\text {max }}\right]}(\dot{\hat{\tau}})$ then, for all $t>0$, one has

$$
\hat{\tau}_{p}(t) \in\left[\tau_{\min }, \tau_{\max }\right]
$$

where $\tau_{\min }$ and $\tau_{\max }$ are defined in Assumption 3.

The proof is given in [31]. In the sequel, it will be assumed that the following Assumption 6 holds.

\section{Assumption 6}

The initial condition of the estimated delay is such that

$$
\hat{\tau}(0) \in\left[\tau_{\min }, \tau_{\max }\right] .
$$

\section{Remark 3.2}

With a slight abuse of notation, the projected delay $\hat{\tau}_{p}$ will be denoted $\hat{\tau}$ in the sequel. It was shown in [32] that the projection retains the properties that are established in absence of the projection and guarantees that (12) is verified. As a result, the error $\hat{\tau}(t)-\tau(t)$ is bounded for all $t>0$. 
The following notations will be used in the next computations $\hat{\Lambda}_{\text {ext }}=$ $\Lambda_{\text {ext }}(\hat{\xi}(t))$ and $\Lambda_{\text {ext }}=\Lambda_{\text {ext }}(\xi(t))$. Denoting by $e=\hat{X}-X$ the estimation error, its dynamics is governed by

$$
\dot{e}=\hat{\Lambda}_{e x t} \hat{X}-\Lambda_{e x t} X+f_{e x t}(\hat{X})-f_{e x t}(X)-\lambda^{-1}(\hat{\xi}) S_{\rho}^{-1} C_{e x t}^{T} C_{e x t} e-\Gamma(t, y, \tau)
$$

For the proof of the main result, the next assumption is required:

\section{Assumption 7}

There are time intervals $I$ where $\hat{\xi}$ is bounded from below and its time-derivative is bounded, i.e. for all $t \in I$

$$
\alpha \leq|\hat{\xi}(t)| \quad \text { and } \quad|\dot{\hat{\xi}}(t)| \leq \nu
$$

with $\alpha, \nu>0$.

\section{Remark 3.3}

Inequality $|\dot{\hat{\xi}}(t)| \leq \nu$ is a technical condition that will be used in the next section to prove the convergence of the estimation error; this condition arises because of the choice of the high gain observer. Note that, this condition will be usually verified in practice because all real signals are bounded. Inequality $\alpha \leq|\hat{\xi}(t)|$ appears because of the form of the extended system (8) and is linked to the intrinsic observability conditions $G(y) \neq 0$ and $\dot{u} \neq 0$ presented in Theorem 1 .

It is now possible to state the main result of our article.

\section{Theorem 2}

Let $I=\left[t_{0}, t\right]$ with $t_{0}>0$ such that Assumptions 1, 2, 5, 4, 6 and 7 hold on $I$. Then, provided that $\rho$ is sufficiently large, there exist $k, \sigma, r>0$ such that for all $t \in I$

$$
\|e(t)\| \leq k\left\|e\left(t_{0}\right)\right\| e^{-\sigma\left(t-t_{0}\right)}+r .
$$

\section{Proof}

The proof use similar argument as in [29]. First note that $\Lambda_{e x t}=\lambda^{-1}(\xi) \bar{A} \lambda(\xi)$ and $C_{\text {ext }} \lambda(\xi)=C_{\text {ext }}$. Furthermore,

$$
\hat{\Lambda}_{e x t} \hat{X}-\Lambda_{e x t} X=\left(\hat{\Lambda}_{e x t}-\Lambda_{e x t}\right) \hat{X}+\Lambda_{e x t} e
$$

and the only non zero term of $\left[\hat{\Lambda}_{e x t}-\Lambda_{e x t}\right] \hat{X}$ is

$$
\begin{aligned}
\left(\left[\hat{\Lambda}_{\text {ext }}-\Lambda_{\text {ext }}\right] \hat{X}\right)_{(n, 1)} & =G(y)\left[\sum_{i=1}^{m} \frac{(-1)^{i} \hat{\tau}^{i-1}}{i !} u^{(i)}(t)-\sum_{i=1}^{m} \frac{(-1)^{i} \tau^{i-1}}{i !} u^{(i)}(t)\right] \hat{\tau} \\
& =\hat{\tau} G(y) \sum_{i=1}^{m} \frac{(-1)^{i}}{i !} u^{(i)}(t)\left[\hat{\tau}^{i-1}-\tau^{i-1}\right] .
\end{aligned}
$$


Since all the terms on the right hand side of (18) are bounded then

$$
\left\|\left(\left[\hat{\Lambda}_{e x t}-\Lambda_{e x t}\right] \hat{X}\right)_{(n, 1)}\right\| \leq c_{0}
$$

with $c_{0}>0$. In addition, since $f$ is $C^{1}$ according to Assumption 1 then $f_{\text {ext }}$ is also $C^{1}$ and from the mean value theorem one gets

$$
f_{\text {ext }}(\hat{X})-f_{\text {ext }}(X)=\left.\frac{d f_{\text {ext }}(X)}{d X}\right|_{\kappa} e
$$

for some $\kappa$ lying between the segment with end points $X$ and $\hat{X}$. Besides, the Jacobian matrix of $f_{\text {ext }}$ is lower triangular from the definition of $f$. Substituting (17) and (20) in (14) gives

$$
\dot{e}=\left(\Lambda_{e x t}-\lambda^{-1}(\hat{\xi}) S_{\rho}^{-1} C_{e x t}^{T} C_{e x t}\right) e+\left(\hat{\Lambda}_{e x t}-\Lambda_{e x t}\right) \hat{X}+\left.\frac{d f_{e x t}(X)}{d X}\right|_{\kappa} e-\Gamma(t, y, h) .
$$

Let $\Delta_{\rho}$ be the diagonal matrix

$$
\Delta_{\rho}=\operatorname{diag}\left(1, \frac{1}{\rho}, \ldots, \frac{1}{\rho^{n}}\right) \in \mathbb{R}^{(n+1) \times(n+1)}
$$

then it is shown in [29] that $S_{\rho}=\frac{1}{\rho} \Delta_{\rho} S_{1} \Delta_{\rho}$ where $S_{1}$ is the solution of the Lyapunov equation in (9) for $\rho=1$. Now, set $\bar{e}=\lambda(\hat{\xi}) \Delta_{\rho} e$, then

$$
\begin{aligned}
\dot{\bar{e}}= & \rho\left(\bar{A}-S_{1}^{-1} C_{e x t}^{T} C_{e x t}\right) \bar{e}+\lambda(\hat{\xi}) \Delta_{\rho}\left(\hat{\Lambda}_{e x t}-\Lambda_{e x t}\right) \hat{X} \\
& +\left.\lambda(\hat{\xi}) \Delta_{\rho} \frac{d f_{e x t}(X)}{d X}\right|_{\kappa} \Delta_{\rho}^{-1} \lambda^{-1}(\hat{\xi}) \bar{e} \\
& +\dot{\lambda}(\hat{\xi}) \lambda^{-1}(\hat{\xi}) \bar{e}-\lambda(\hat{\xi}) \Delta_{\rho} \Gamma(t, y, h) .
\end{aligned}
$$

Furthermore, $\lambda(\hat{\xi}) \Delta_{\rho} \frac{d f_{e x t}(x)}{d X} \Delta_{\rho}^{-1} \lambda^{-1}(\hat{\xi})=\Delta_{\rho} \frac{d f_{e x t}(x)}{d X} \Delta_{\rho}^{-1}$ and we have

$$
\Delta_{\rho} \frac{d f_{e x t}(x)}{d X} \Delta_{\rho}^{-1}=\left[\begin{array}{ccccc}
\frac{\partial f_{1}}{\partial x_{1}} & 0 & \ldots & 0 & 0 \\
\frac{1}{\rho} \frac{\partial f_{2}}{\partial x_{1}} & \frac{\partial f_{2}}{\partial x_{2}} & \ddots & \vdots & 0 \\
\vdots & \ddots & \ddots & 0 & \vdots \\
\frac{1}{\rho^{n-1}} \frac{\partial f_{n}}{\partial x_{1}} & \ldots & \frac{1}{\rho} \frac{\partial f_{n}}{\partial x_{n-1}} & \frac{\partial f_{n}}{\partial x_{n}} & 0 \\
0 & 0 & 0 & \ldots & 0
\end{array}\right] .
$$

Since the partial derivative of the $f_{i}$ are bounded and choosing $\rho \geq 1$, there exists a constant $c_{0}$ independent from $\rho$ such that

$$
\left\|\left.\lambda(\hat{\xi}) \Delta_{\rho} \frac{d f_{\text {ext }}(X)}{d X}\right|_{\kappa} \lambda^{-1}(\hat{\xi}) \Delta_{\rho}^{-1}\right\| \leq c_{1} .
$$


Besides, the product $\lambda(\hat{\xi}) \Delta_{\rho}\left(\hat{\Lambda}_{e x t}-\Lambda_{e x t}\right) \hat{X}$ results in a matrix whose coefficients are equal to zero except one that depends on $\left(\left[\hat{\Lambda}_{e x t}-\Lambda_{e x t}\right] \hat{X}\right)_{(n, 1)}$ so one gets

$$
\left\|\lambda(\hat{\xi}) \Delta_{\rho}\left(\hat{\Lambda}_{e x t}-\Lambda_{e x t}\right) \hat{X}\right\| \leq \frac{c_{0}}{\rho} .
$$

From Assumption 7, one derives that $\left\|\dot{\lambda}(\hat{\xi}) \lambda^{-1}(\hat{\xi})\right\| \leq \frac{\nu}{\alpha}$ for all $t \in I$. Define the following Lyapunov candidate function $V$ as follows

$$
V(t)=\bar{e}(t)^{T} S_{1} \bar{e}(t)
$$

The matrix $S_{1}$ verifies

$$
\underline{\delta}_{1}\|\bar{e}\|^{2} \leq \bar{e}^{T} S_{1} \bar{e} \leq \bar{\delta}_{1}\|\bar{e}\|^{2}
$$

with $\underline{\delta}_{1}, \bar{\delta}_{1}>0$ because it is the solution of the Lyapunov equation for $\rho=1$ (see [28]) so the Lyapunov function (27) is well defined. Note that $\left\|\lambda(\hat{\xi}) \Delta_{\rho}\right\| \leq 1$ for $\rho \geq 1$. Differentiating $V$ along the trajectories of (23) and using above properties leads to

$$
\begin{aligned}
\dot{V} \leq & -\rho \bar{e}^{T} S_{1} \bar{e}+2 \frac{c_{0}}{\rho}\left\|S_{1}\right\|\|\bar{e}\|+2 c_{1}\left\|S_{1}\right\|\|\bar{e}\|^{2} \\
& +2 \frac{\nu}{\alpha}\left\|S_{1}\right\|\|\bar{e}\|^{2}+2\left\|S_{1}\right\|\|\Gamma(t, y, \tau)\|\|\bar{e}\| .
\end{aligned}
$$

Thus, the following maximization of (29) is obtained

$$
\dot{V} \leq-\left(\rho \underline{\delta}_{1}-2 c_{1}\left\|S_{1}\right\|-2 \frac{\nu}{\alpha}\left\|S_{1}\right\|\right)\|\bar{e}\|^{2}+2\left\|S_{1}\right\|\left(\frac{c_{0}}{\rho}+\|\Gamma(t, y, \tau)\|\right)\|\bar{e}\| .
$$

In addition, from equation (7) and Assumptions 2, 3 and 4, it can be deduced that $\Gamma$ is bounded and verifies $\sup _{t \geq 0}\|\Gamma(t, y, h)\|<\bar{\Gamma}$ with $\bar{\Gamma}=\bar{G} \frac{\tau_{m a x}^{m+1}}{(m+1) !} U_{m+1}+\bar{\eta}$. Consequently, the gain $\rho$ can be chosen sufficiently large such that the above inequality becomes

$$
\dot{V}(t) \leq-c_{3} V(t)+c_{4} \sqrt{V(t)}
$$

with $c_{3}=\left(\rho \underline{\delta}_{1}-2 c_{1}\left\|S_{1}\right\|-2 \frac{\nu}{\alpha}\left\|S_{1}\right\|\right) / \bar{\delta}_{1}>0$ and $c_{4}=2\left\|S_{1}\right\|\left(\frac{c_{0}}{\rho}+\bar{\Gamma}\right) / \sqrt{\underline{\delta}_{1}}$. The comparison lemma given in [33, Lemma 9.4] and the relations $\bar{e}=\lambda(\hat{\xi}) \Delta_{\rho} e$ and $e=\Delta_{\rho}^{-1} \lambda^{-1}(\hat{\xi}) \bar{e}$ ensure that equation (16) is satisfied.

\section{Remark 3.4}

Theorem 2 can be extended to systems such that $G$ depends on $x$ provided the extra assumption $G$ globally Lipschitz is made.

\section{Remark 3.5}

As it has been mentioned before, in practice, an accurate estimation can be obtained with only the first two derivatives of the input. To compute these derivatives, it may be necessary to use a numerical differentiator. In this case, inevitable errors are introduced by the differentiator. Denoting $\hat{u}^{(i)}$ the i-th estimated time derivatives and $e_{u_{i}}=u^{(i)}-\hat{u}^{(i)}$, one obtains that $u^{(i)}=e_{u_{i}}+\hat{u}^{(i)}$ so the Taylor approximation (6) 
becomes

$$
u(t-\tau(t))=\sum_{i=0}^{m} \frac{(-1)^{i} \tau(t)^{i}}{i !} \hat{u}^{(i)}(t)+\gamma_{m}^{\prime}(t-\tau(t))
$$

with $\gamma_{m}^{\prime}(t,-\tau(t))=\gamma_{m}(t,-\tau(t))+\sum_{i=0}^{m} \frac{(-1)^{i} \tau(t)^{i}}{i !} e_{u_{i}}(t)$. If the $e_{u_{i}}$ are bounded and sufficiently small then $\gamma_{m}^{\prime}$ is bounded and the extra error added by the numerical differentiation will decrease the observation accuracy by increasing the convergence radius $r$ in (16). The effect of input noise can also be included in the differentiation error.

\section{Remark 3.6}

If some noise affects the output $y$, a trade-off between noise amplification and accuracy of the estimation will be necessary. Indeed, it can be shown that the radius $r$ decreases when $\rho$ increases, however, it is well known as explained in [28] that the high-gain observer amplifies the noise if the gain is large.

\section{SIMULATION}

In this section, the performances of the observer will be illustrated by some simulations.

\subsection{Example 1}

A pendulum system has been chosen to illustrate previous results. Its representation reads as

$$
\left\{\begin{array}{l}
\dot{x}(t)=\left[\begin{array}{c}
x_{2} \\
-\frac{g}{l} \sin x_{1}-\frac{k}{m_{p}} x_{2}
\end{array}\right]+\left[\begin{array}{c}
0 \\
\frac{1}{m_{p} l^{2}}
\end{array}\right] u(t-\tau(t)) \\
y(t)=C x(t)=x_{1}(t) .
\end{array}\right.
$$

where $g=9.81 \mathrm{~m} \cdot \mathrm{s}^{-2}$ is the acceleration due to gravity, $m_{p}=0.2 \mathrm{~kg}$ is the mass of the bob, $l=0.5 \mathrm{~m}$ is the length of the rod and $k=0.3 \mathrm{~kg} . \mathrm{s}^{-1}$ is the friction coefficient. Consequently, (33) has the form of (1) with $\Lambda=\left[\begin{array}{ll}0 & 1 \\ 0 & 0\end{array}\right], F(x)=-\frac{g}{l} \sin x_{1}-\frac{k}{m_{p}} x_{2}$ and $G(y)=\frac{1}{m_{p} l^{2}}$. The functions $f$ and $G$ satisfy Assumptions 1 and 2 .

The delays used in the sequel are defined by $\tau(t)=0.6$ for $t \in[0,20[, \tau(t)=1.2$ for $t \in[20,40[, \tau(t)=0.3$ for $t \in[40,60[$ and $\tau(t)=0.8+0.45 \sin (0.2 t)$ for $t \geq 60$.

Thus, $\tau$ is bounded in $\left[\tau_{\min }, \tau_{\max }\right]$ with $\tau_{\min }=0 \mathrm{~s}$ and $\tau_{\max }=1.5 \mathrm{~s}$. The delay is differentiable on $[60,120]$ so Assumption 3 is verified. The observability condition (3) is always true since $G$ is constant.

In above sections, the general case of the Taylor expansion of order $m$ has been studied. In practice, the choice of $m$ can be made according to the precision desired on $\hat{\tau}$ and the available time derivatives of $u$. In practice the computation of the input time derivatives for $m>2$ is challenging especially if some noise is present. That is why in this section, two observers are going to be tested with $m=1, m=2$. For both observers, the gain $\rho$ 
is equal to 5 . It will be shown that $m=2$ is sufficient to get an accurate estimation using a limited number of input differentiation. The observer equations are given below:

$$
\left\{\begin{aligned}
\dot{\hat{X}}= & \hat{\Lambda}_{e x t} \hat{X}+\left[0,-\frac{g}{l} \sin \hat{x}_{1}-\frac{k}{m_{p}} \hat{x}_{2}, 0\right]^{T} \\
& +\left[0, \frac{1}{m_{p} l^{2}}, 0\right]^{T} u(t)-\lambda^{-1}(\hat{\xi}) S_{\rho}^{-1}\left(\hat{x}_{1}-x_{1}\right) \\
0= & -\rho S_{\rho}-\bar{A}^{T} S_{\rho}-S_{\rho} \bar{A}+C_{e x t}^{T} C_{e x t} \\
\dot{\hat{\tau}}_{p}= & \operatorname{proj}_{\left[\tau_{\text {min }}, \tau_{\text {max }}\right]}(\dot{\hat{\tau}})
\end{aligned}\right.
$$

where $\hat{X}=\left[\hat{x}_{1}, \hat{x}_{2}, \hat{\tau}\right]^{T}, \hat{\Lambda}_{\text {ext }}$ and $\bar{A}$ are defined at the beginning of Section 3.2 and with

$$
\hat{\xi}= \begin{cases}-\frac{1}{m_{p} l^{2}} \dot{u} & \text { for } m=1, \\ \frac{1}{m_{p} l^{2}}\left(-\dot{u}+\frac{1}{2} \ddot{u} \hat{\tau}\right) & \text { for } m=2,\end{cases}
$$

and $C_{\text {ext }}=[1,0,0]$. The initial conditions of the system and of the observer are gathered in Table I.

Table I. Observer parameter and initial conditions

\begin{tabular}{c|c|c|c|c}
\hline$x(0)\left[\mathrm{rad}, \mathrm{rad} . \mathrm{s}^{-1}\right]$ & $\hat{x}(0)\left[\mathrm{rad}, \mathrm{rad} . \mathrm{s}^{-1}\right]$ & $\hat{\tau}(0)(\mathrm{s})$ & $\rho$ & $S(0)$ \\
\hline$[0.87,0]^{T}$ & {$[0,0]^{T}$} & 0.3 & 5 & $I_{3}$ \\
\hline
\end{tabular}

On Figure 1, the input $u(t)=0.009 t$ is applied to the system. This input is smooth and Assumption 4 is satisfied. In addition, condition (4) is true because $\dot{u}(t)=0.009 \neq 0$ so there is no observation singularity. Since $\ddot{u}(t)=0$, one can see from (35) that $\hat{\xi}$ has the same value $\left(\hat{\xi}=-\frac{1}{m_{p} l^{2}} 0.009\right)$ for $m=1$ and $m=2$ that is why the curves are exactly mixed up on Figure 1. In addition $\hat{\xi}$ is always different from zero so Assumption 7 is true. For this ideal case, the convergence is asymptotic for a constant delay $(t \in[0,60])$ and the convergence radius can be reduced arbitrarily by increasing the observer gain $\rho$ for the time-varying delay case $(t \in[60,120])$. In order to show the advantage of estimating the delay to have a more accurate state estimation, we have implemented the standard high-gain observer defined by

$$
\dot{\hat{x}}(t)=\left[\begin{array}{c}
\hat{x}_{2} \\
-\frac{g}{l} \sin \hat{x}_{1}-\frac{k}{m_{p}} \hat{x}_{2}
\end{array}\right]+\left[\begin{array}{c}
0 \\
\frac{1}{m_{p} l^{2}}
\end{array}\right] u\left(t-\tau_{m}\right)-S_{\rho}^{-1}\left(\hat{x}_{1}-x_{1}\right)
$$

where $\tau_{m}$ is an average value of the delay taken equal to 0.8 . It is clear from Figure 1 that the state estimation is more accurate using the high-gain observer that also estimates the delay (observer (34)) than standard high-gain observer (36).

On Figure 2, $u(t)=\sin (0.2 t)$ which is smooth and satisfies Assumption 4 is applied to system (33). Condition (4) is true almost everywhere (except when $0.15 t=\pi / 2[2 \pi]$ ). Note that one solution to limit the effect of the singularity in the observer is to "switch off" the observer gain when $|\hat{\xi}|$ gets close to zero. On Figure 2, the observer is arbitrarily switched off for $|\hat{\xi}|<0.7$. In practice, noise can affect the measurement and the input. In this simulation, white noise has been added to the measured signal $x_{1}$ and the input signal $u$. The variance has been chosen to obtain a noise of about $5 \%$ of the amplitude of 

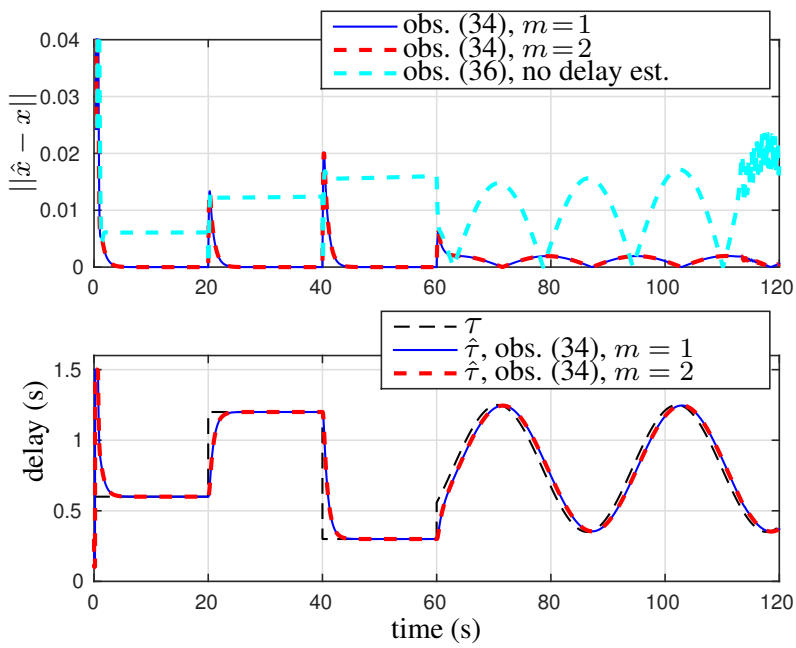

Figure 1. $u(t)=0.009 t, \rho=5$, no noise

the original signals. The time derivatives $\dot{u}, \ddot{u}$ have been computed thanks to the Levant differentiator [34] given by

$$
\left\{\begin{array}{l}
\dot{z}_{0}=v_{0} \\
v_{0}=-\lambda_{0} L^{\frac{1}{3}}\left|z_{0}-u(t)\right|^{\frac{2}{3}} \operatorname{sign}\left(z_{0}-u(t)\right)+z_{1} \\
\dot{z}_{1}=v_{1} \\
v_{1}=-\lambda_{1} L^{\frac{1}{2}}\left|z_{1}-v_{0}\right|^{\frac{1}{2}} \operatorname{sign}\left(z_{1}-v_{0}\right)+z_{2} \\
\dot{z}_{2}=-\lambda_{2} \operatorname{sign}\left(z_{2}-v_{1}\right) .
\end{array}\right.
$$

If the parameters $\lambda_{i}$ and $L$ are properly chosen, then [34, Theorem 5] guarantees that the following equalities

$$
z_{0}(t)=u(t) \text { and } z_{i}(t)=u^{(i)}(t) \text { for } i=1,2 .
$$

are true in the absence of input noise and after a finite time. If the input $u$ is noisy then the estimation error converges in a ball around the origin whose radius is proportional to the noise value [34, Theorem 6]. The differentiator parameters are $\lambda_{0}=3, \lambda_{1}=1.5$, $\lambda_{2}=1$ and $L=0.11$.

Figure 2 shows that in spite of the input and measurement noises, the observer is able to estimate both the delay and the state. Note that the peaks of the blue curve $m=1$ are due to the observation singularity when $\dot{u}=0$ that implies $\hat{\xi}=0$. Increasing $m$ allows to reduce the effect of the singularity by using the higher derivatives. Note also that in the case of a noisy measurement it is not possible to reduce arbitrarily the estimation error by increasing the gain. Indeed, the noise will be amplified if the gain is too large as mentioned in Remark 3.6.

\section{Remark 4.1}

The choice of the input $u(t)=\sin (0.2 t)$ is made in order to ensure that the input does not vary too fast with respect to the delay size. Indeed, this implies that the Taylor's 
expansion is accurate and that the delay can be correctly estimated. If the delay is smaller then the input frequency can be increased without degrading the estimation accuracy.
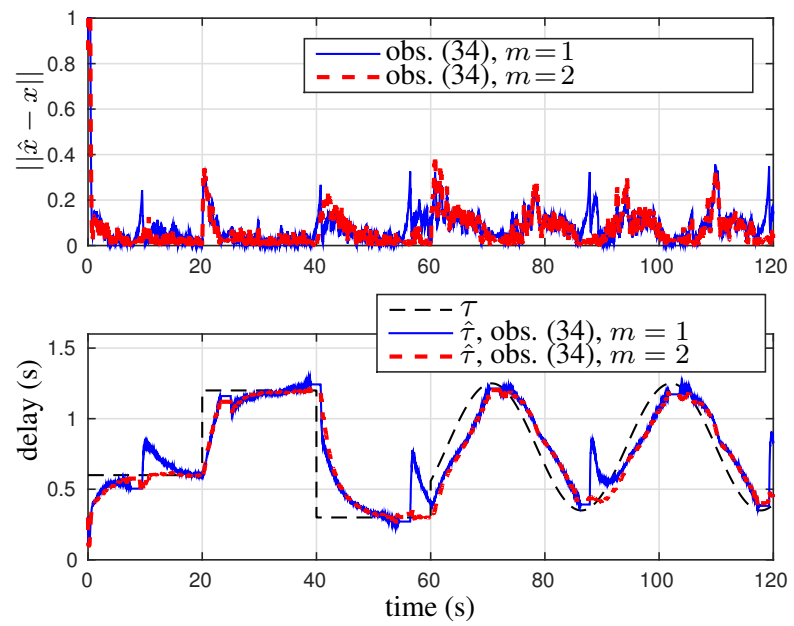

Figure 2. $u(t)=\sin (0.2 t), \rho=2.5$, with noise and observer switched off for $|\hat{\xi}|<0.7$

\subsection{Example 2}

In order to compare our method with existing delay estimation techniques, we provide a second example. Since existing methods that estimate the input delay only focus on linear systems with known states, we chose the following linear scalar system:

$$
\dot{x}(t)=-x(t)+u(t-\tau(t))
$$

where we assume that $x(t)$ is known and $\tau(t)$ is the same as in Example 1. Figure 3 shows a comparison of our method with the delay estimators presented in [9], [12], [26]. It is clear that the methods of [9], [26] only work for a constant input delay. The method in [12] tracks accurately the delay variation but exhibits oscillations when the delay is constant (the magnitude of these oscillations is larger for a large delay). The delay estimation using our extended observer method tracks accurately the delay and do not exhibit oscillation for a constant delay. Note that the estimator used in [12] seems more accurate when the delay is time-varying. In order to qualify this analysis, it has not to be forgotten that the full knowledge of the state and its time-derivative is required in [12]. In addition, the results in [12] are not applicable to nonlinear systems.

\section{CONCLUSION}

In this article, a new solution based on observation techniques is presented to reconstruct both the delay and the state of a class of nonlinear SISO systems. The method works 


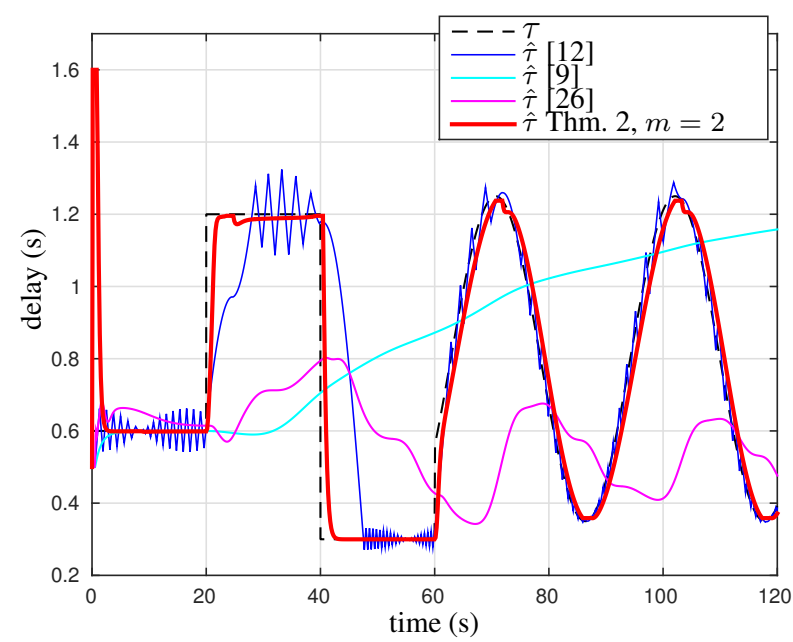

Figure 3. Comparison of different input delay estimation methods, $u(t)=\sin (0.2 t)$, no noise

for time-varying delays. The Taylor expansion is exploited to take the delay out of the retarded input. Then, the initial system is turned into an extended system with the delay as a part of the augmented state. An observability condition is derived from the analysis of this extended system. Finally, a high-gain observer is designed and the convergence ot the estimation error in a neighborhood of the origin is proved. All the results are illustrated by numerous simulations. The extension to nonlinear MIMO systems as well as the observation in closed-loop are considered for future developments.

\section{REFERENCES}

1. Gao H, Chen T, Lam J. A new delay system approach to network-based control. Automatica 2008; 44(1):39-52.

2. Smith OJM. Closer control of loops with dead time. Chemical Engineering Progress 1957; 53(5):217-219.

3. O'Dwyer A. A survey of techniques for the estimation and compensation of processes with time delay. Technical Report AOD.00.03, Dublin Institute of technology 2000.

4. Clifford G. Time delay estimation for passive sonar signal processing. IEEE Transactions on Acoustics, Speech and Signal Processing 1981; 29(3):463-470.

5. Knapp C, Carter GC. The generalized correlation method for estimation of time delay. IEEE Transactions on Acoustics, Speech and Signal Processing 1976; 24(4):320-327.

6. Jacovitti G, Scarano G. Discrete time techniques for time delay estimation. IEEE Transactions on Signal Processing 1993; 41(2):525-533.

7. Björklund S. A survey and comparison of time-delay estimation methods in linear systems. $\mathrm{PhD}$ Thesis, Linköping University, Sweden 2003.

8. Agarwal M, Canudas C. On-line estimation of time delay and continuous-time process parameters. American Control Conference, Seattle, USA, 1986.

9. Tuch J, Feuer A, Palmor ZJ. Time delay estimation in continuous linear time-invariant systems. IEEE Trans. Autom. Control 1994; 39(4):823-827.

10. Nihtilä M, Damak T, Babary JP. On-line estimation of the time delay via orthogonal collocation. Simulation Practice and Theory 1997; 5(2):101-120.

11. Diop S, Kolmanovsky I, Moraal PE, van Nieuwstadt M. Preserving stability-performance when facing an unknown time-delay. Control Engineering Practice 2001; 9(12):1319-1325. 
12. Drakunov SV, Perruquetti W, Richard JP, Belkoura L. Delay identification in time-delay systems using variable structure observers. Annual Reviews in Control 2006; 30(2):143-158.

13. Belkoura L, Richard JP, Fliess M. A convolution approach for delay systems identification. 17th IFAC World Congress, Seoul, South Korea, 2008.

14. Belkoura L, Richard JP, Fliess M. Parameters estimation of systems with delayed and structured entries. Automatica 2009; 45(5):1117-1125.

15. Bresch-Pietri D, Krstic M. Delay-adaptive predictor feedback for systems with unknown long actuator delay. IEEE Trans. Autom. Control 2010; 55(9):2106-2112.

16. Gaudette DL, Miller DE. Stabilizing a SISO LTI plant with gain and delay margins as large as desired. IEEE Trans. Autom. Control 2014; 59(9):2324-2339.

17. Sename O. New trends in design of observers for time-delay systems. Kybernetika 2001; 37(4):427458.

18. Ghanes M, de León Morales J, Barbot JP. Observer design for nonlinear systems under unknown time-varying delays. IEEE Trans. Autom. Control 2013; 58(6):1529-1534.

19. Cacace F, Germani A, Manes C. An observer for a class of nonlinear systems with time varying observation delay. Systems \& Control Letters 2010; 59(5):305-312.

20. Farza M, Sboui A, Cherrier E, M'Saad M. High-gain observer for a class of time-delay nonlinear systems. International Journal of Control 2010; 83(2):273-280.

21. Ghanes M, de León J, Barbot JP. Simultaneous observation and identification for nonlinear systems under unknown time-varying delays. Journal of the Franklin Institute 2016; 353(10):2305-2318.

22. Cacace F, Conte F, Germani A, Palombo G. Delay identification for a class of nonlinear systems. International Journal of Control 2016; 89(11):2350-2359.

23. Léchappé V, De León J, Moulay E, Plestan F, Glumineau A. Delay and state observer for SISO LTI systems. American Control Conference, Chicago, USA, 2015.

24. Léchappé V, Moulay E, Plestan F, Glumineau A. Interconnected delay and state observer for nonlinear system with input delay. American Control Conference, Boston, USA, 2016.

25. Gauthier JP, Bornard G. Observability for any $u(t)$ of a class of nonlinear systems. IEEE Trans. Autom. Control 1981; 26(4):922-926.

26. Na J, Ren X, Xia Y. Adaptive parameter identification of linear SISO systems with unknown timedelay. Systems \& Control Letters 2014; 66(0):43-50.

27. Hermann R, Krener AJ. Nonlinear controllability and observability. IEEE Trans. Autom. Control 1977; 22(5):728-740.

28. Gauthier JP, Hammouri H, Othman S. A simple observer for nonlinear systems applications to bioreactors. IEEE Trans. Autom. Control 1992; 37(6):875-880.

29. Busawon KK, Farza M, Hammouri H. Observer design for a special class of nonlinear systems. International Journal of Control 1998; 71(3):405-418.

30. Busawon KK, de León Morales J. An observer design for uniformly observable non-linear systems. International Journal of Control 2000; 73(15):1375-1381.

31. Lavretsky E, Gibson TE, Annaswamy AM. Projection operator in adaptive systems 2011; ArXiv:1112.4232.

32. Ioannou PA, Sun J. Robust adaptive control. Dover Books on Electrical Engineering, Dover Publication, 2012.

33. Khalil HK. Nonlinear Systems. $3^{\text {rd }}$ edn., Prentice Hall, 2002.

34. Levant A. Sliding order and sliding accuracy in sliding mode control. International Journal of Control 1993; 58(6):1247-1263. 